\title{
Some Extensions of W. Gautschi's Inequalities for the Gamma Function
}

\author{
By D. Kershaw
}

Abstract. It has been shown by W. Gautschi that if $0<s<1$, then for $x \geqslant 1$

$$
x^{1-s}<\frac{\Gamma(x+1)}{\Gamma(x+s)}<\exp [(1-s) \psi(x+1)] .
$$

The following closer bounds are proved:

$$
\exp \left[(1-s) \psi\left(x+s^{1 / 2}\right)\right]<\frac{\Gamma(x+1)}{\Gamma(x+s)}<\exp \left[(1-s) \psi\left(x+\frac{s+1}{2}\right)\right]
$$

and

$$
\left[x+\frac{s}{2}\right]^{1-s}<\frac{\Gamma(x+1)}{\Gamma(x+s)}<\left[x-\frac{1}{2}+\left(s+\frac{1}{4}\right)^{1 / 2}\right]^{1-s} .
$$

These are compared with each other and with inequalities given by T. Erber and J. D. Kečkic and P. M. Vasic.

1. Introduction and Summary. It was shown by W. Gautschi in [4] that $\Gamma$, the gamma function, satisfies the following inequalities:

$$
n^{1-s}<\frac{\Gamma(n+1)}{\Gamma(n+s)}<\exp [(1-s) \psi(n+1)]
$$

for $0<s<1$ and $n=1,2, \ldots$, where $\psi=\Gamma^{\prime} / \Gamma$, the logarithmic derivative of the gamma function. The main purpose of this note is to give proofs of the following closer bounds:

$$
\exp \left[(1-s) \psi\left(x+s^{1 / 2}\right)\right]<\frac{\Gamma(x+1)}{\Gamma(x+s)}<\exp \left[(1-s) \psi\left(x+\frac{s+1}{2}\right)\right]
$$

and

$$
\left[x+\frac{s}{2}\right]^{1-s}<\frac{\Gamma(x+1)}{\Gamma(x+s)}<\left[x-\frac{1}{2}+\left(s+\frac{1}{4}\right)^{1 / 2}\right]^{1-s},
$$

each being valid for $x>0$ and $0<s<1$.

Other bounds have been found for the same quotient by Erber [2] and Kečkić and Vasic [5]. Comparisons are made with these and with each other.

2. Proofs of Inequalities. Define the functions $f$ and $g$ by

$$
f(x)=\frac{\Gamma(x+1)}{\Gamma(x+s)} \cdot \exp [(s-1) \psi(x+\alpha)]
$$

Received November 30, 1982.

1980 Mathematics Subject Classification. Primary 33A15, 26 D20.

Key words and phrases. Gamma function, inequalities. 
and

$$
g(x)=\frac{\Gamma(x+1)}{\Gamma(x+s)} \cdot(x+\beta)^{s-1}
$$

for $x>0$ and $0<s<1$, where the parameters $\alpha$ and $\beta$ are to be determined. It is not difficult to show, with the aid of Stirling's formula, that

$$
\lim _{x \rightarrow \infty} f(x)=\lim _{x \rightarrow \infty} g(x)=1 .
$$

Now let

$$
F(x)=\frac{f(x)}{f(x+1)}=\left(\frac{x+s}{x+1}\right) \cdot \exp \left(\frac{1-s}{x+\alpha}\right) .
$$

Then

$$
\frac{F^{\prime}(x)}{F(x)}=(1-s) \frac{\left(\alpha^{2}-s\right)+(2 \alpha-s-1) x}{(x+1)(x+s)(x+\alpha)^{2}} .
$$

It is easy to show that

(a) if $\alpha=s^{1 / 2}$, then $F^{\prime}(x)<0$ for $x>0$

and
(b) if $\alpha=\frac{1}{2}(s+1)$, then $F^{\prime}(x)>0$ for $x>0$.

Consequently if $\alpha=s^{1 / 2}$ then $F$ strictly decreases, and since $F(x) \rightarrow 1$ as $x \rightarrow \infty$ it follows that

$$
F(x)>1 \text { for } x>0 .
$$

But, from (2.3), this implies that

$$
f(x)>f(x+1) \text { for } x>0
$$

and so

$$
f(x)>f(x+n) .
$$

Take the limit as $n \rightarrow \infty$ to give the result that $f(x)>1$, which can be rewritten as

$$
\frac{\Gamma(x+1)}{\Gamma(x+s)}>\exp \left[(1-s) \psi\left(x+s^{1 / 2}\right)\right]
$$

which is the lower bound in (1.2). The corresponding upper bound can be verified by a similar argument when $\alpha=\frac{1}{2}(s+1)$, the only difference being that in this case $f$ strictly increases to unity.

To prove the inequalities of (1.3) define

$$
G(x)=\frac{g(x)}{g(x+1)}=\left(\frac{x+s}{x+1}\right)\left(\frac{x+\beta+1}{x+\beta}\right)^{1-s},
$$

from which it follows that

$$
\frac{G^{\prime}(x)}{G(x)}=\frac{(1-s)\left[\left(\beta^{2}+\beta-s\right)+(2 \beta-s) x\right]}{(x+1)(x+s)(x+\beta)(x+\beta+1)} .
$$

This will lead to

$$
\text { (c) if } \beta=\frac{1}{2} s \text {, then } G^{\prime}(x)<0 \text { for } x>0 \text {, }
$$


and

(d) if $\beta=-\frac{1}{2}+\left(s+\frac{1}{4}\right)^{1 / 2}$, then $G^{\prime}(x)>0$ for $x>0$.

The same arguments which were used on $F$ can now be used on $G$ to give the inequalities (1.3).

3. Comparisons. The relative merits of the bounds of (1.2) and (1.3) are not obvious. However it can be shown that

$$
\psi\left(x+s^{1 / 2}\right)-\log (x+s / 2)
$$

changes sign in the interval $0<s<1$, and so neither lower bound is best overall. It has not been possible to prove a similar result for

$$
\psi(x+(s+1) / 2)-\log \left[x-\frac{1}{2}+\left(s+\frac{1}{4}\right)^{1 / 2}\right],
$$

but numerical calculations show that this also changes sign in $0<s<1$. Consequently it seems likely that neither (1.2) nor (1.3) can be declared the outright winner.

4. Erber's Inequality. The following result was given by T. Erber in [2]:

$$
\frac{\Gamma(n+1)}{\Gamma(n+s)}<\frac{4(n+s)}{4 n+(s+1)^{2}}(n+1)^{1-s}, \quad 0<s<1, n=1,2, \ldots
$$

We shall show that the upper bound in (3.7) is an improvement over this, which will be the case if

$$
\left[n+\frac{s+1}{2}\right]^{1} s<\frac{4(n+s)}{4 n+(s+1)^{2}} \cdot(n+1)^{1-s}
$$

This can be rearranged as

$$
\left[1-\frac{1-s}{2 n+2}\right]^{s-1}>1+\frac{(1-s)^{2}}{4(n+s)} .
$$

Now Bernoulli's inequality [1, p. 12], i.e.

$$
(1-x)^{-\alpha}>1+\alpha x \text { for } \alpha>0 \text { and } 0<x<1,
$$

gives

$$
\left[1-\frac{1-s}{2 n+2}\right]^{s-1}>1+\frac{(1-s)^{2}}{2 n+2}
$$

which is obviously greater than the expression on the right-hand side of (4.2) for $0<s<1, n \geqslant 1$. Consequently (3.7) is an improvement over (4.1) and a fortiori so is the upper bound in (3.8).

5. The Inequalities of Kečkić and Vasić. J. D. Kečkić and P. M. Vasić gave in [5] the inequalities

$$
\frac{b^{b-1}}{a^{a-1}} \cdot e^{-(b-a)}<\frac{\Gamma(b)}{\Gamma(a)}<\frac{b^{b-1 / 2}}{a^{a-1 / 2}} \cdot e^{-(b-a)}, \quad 0<a<b .
$$


In the notation of this paper they will be rewritten as

$$
\frac{(x+1)^{x}}{(x+s)^{x+s-1}} e^{-(1-s)}<\frac{\Gamma(x+1)}{\Gamma(x+s)}<\frac{(x+1)^{x+1 / 2}}{(x+s)^{x+s-1 / 2}} \cdot e^{-(1-s)} \text { for } s>0 .
$$

We shall show that if $2 x+s \geqslant 1$ and $0<s<1$, then the lower bound in (1.3) is an improvement over the lower bound in (5.2); since the latter can be written

$$
\exp \left\{\int_{x+s}^{x+1}\left[\log t-\frac{1}{t}\right] d t\right\}
$$

this will be equivalent to proving that

$$
(1-s) \log \left(x+\frac{s}{2}\right)>\int_{x+s}^{x+1}\left[\log t-\frac{1}{t}\right] d t, \quad 2 x+s \geqslant 1,
$$

or, with a change of notation, that

(5.4) $(b-a) \log \left(\frac{a+b-1}{2}\right)>\int_{a}^{b}\left[\log t-\frac{1}{t}\right] d t, \quad 0<a<b, a+b=2$.

To prove (5.4) we note first that since

$$
\frac{d^{2}}{d t^{2}}\left[\log t-\frac{1}{t}\right]<0
$$

then, from [6, p. 14],

$$
\frac{1}{b-a} \int_{a}^{b}\left[\log t-\frac{1}{t}\right] d t<\log \left(\frac{a+b}{2}\right)-\frac{2}{a+b} \text {. }
$$

Hence

$$
\frac{1}{b-a} \int_{a}^{b}\left[\log t-\frac{1}{t}\right] d t-\log \left(\frac{a+b-1}{2}\right)<\log \left(\frac{a+b}{a+b-1}\right)-\frac{2}{a+b}
$$

the right-hand side is

$$
-\log \left(1-\frac{1}{a+b}\right)-\frac{2}{a+b}
$$

which is easily shown to be negative for $a+b \geqslant 2$, thus proving (5.4).

The situation with regard to the upper bounds is not clear. It is not difficult to show that Kečkić and Vasić's upper bound is an improvement over the simplified upper bound in (3.7). However, a comparison with (1.2) has not been found possible in general. The problem here is to decide which is the smaller of

$$
\frac{1}{1-s} \int_{x+s}^{x+1}\left[\log t-\frac{1}{2 t}\right] d t \text { and } \psi\left(x+\frac{s+1}{2}\right) .
$$

In the notation of Kečkić and Vasić the bound in (1.2) will be an improvement over that in (5.1) if the following expression is positive:

$$
\frac{1}{b-a} \int_{a}^{b}\left[\log t-\frac{1}{2 t}\right] d t-\psi\left(\frac{a+b}{b}\right) \text {. }
$$

Write $b-a=\alpha, b+a=2 z$. Then (5.6) becomes

$$
\frac{1}{\alpha} \int_{z-\alpha / 2}^{z+\alpha / 2}\left[\log t-\frac{1}{2 t}\right] d t-\psi(z) \text {. }
$$


When considered as a function of $\alpha$ this expression can be shown to decrease from its value at $\alpha=0$. Consequently it is greater than its value at $\alpha=1$, namely

$$
\int_{z-1 / 2}^{z+1 / 2}\left[\log t-\frac{1}{2 t}\right] d t-\psi(z)
$$

Numerical calculations suggest that this last expression is positive for $z>1.29$ which implies that the upper bound of (1.2) is an improvement over that of (5.2) for $x>0.79$.

It is unfortunate that this has not been proved rigorously, but since (5.2) is probably more convenient in practice, it is perhaps not important.

6. Acknowledgements. The author is grateful to F. W. J. Olver for references [2] and [5], to W. Gautschi for valuable comments on a previous draft of this paper, and to the referees for their advice.

Department of Mathematics

Cartmel College

Bailrigg, Lancaster LA1 4YL. England

1. E. F. Beckenbach \& R. Bellman, Inequalities, 1st. ed. Springer-Verlag. Berlin and New York. 1961.

2. T. ERBER. "The gamma function inequalities of Gurland and Gautschi," Skand. Aktuarietidskr., 1960, 1961, pp. 27-28.

3. A. Erdelyi et Al., Higher Transcendental Functions, Vol. I, McGraw-Hill, New York, 1953.

4. W. GautsChi, "Some elementary inequalities relating to the gamma and incomplete gamma function," J. Math. Phi's., v. 38, 1959. pp. 77-81.

5. J. D. KeČKIC \& P. M. VASIC. "Some inequalities for the gamma function," Publ. Inst. Math. (Beograd) (N.S.), v. 11, 1971, pp. 107-114.

6. D. S. Mitrinovic, Analytic Inequalities, Springer-Verlag. Berlin and New York, 1970. 